\title{
Condiciones geológicas-geotécnicas de la cuenca de Santiago y su relación con la distribución de daños del terremoto del 27F
}

\author{
Geological and geotechnical conditions of the Santiago basin and its relation with the 27F \\ earthquake damage distribution \\ Fecha de entrega: 5 de diciembre 2014 \\ Fecha de aceptación: 14 de diciembre 2015
}

\section{Loreto Vergara ${ }^{1,2}$ y Ramón Verdugo ${ }^{2}$}

\author{
${ }^{1}$ Departamento de Ingeniería Civil, Universidad de Chile, Blanco Encalada 2002, Santiago, Chile \\ ${ }^{2}$ CMGI Ltda. Av. Lib. Bdo. O’Higgins 2356 Of. D, Santiago, Chile, lvergara@cmgi.cl, rverdugo@cmgi.cl
}

El terremoto del $27 F$ de magnitud $M w=8.8$, afectó a una extensa área del país y dejó una cifra estimada de más de 2 millones de damnificados. En la Región Metropolitana, los daños más graves se centraron en viviendas antiguas, no obstante, también se presentaron daños importantes en construcciones modernas. Con el objetivo de identificar las variables geológicas y geotécnicas de la cuenca de Santiago que influyeron en su respuesta sísmica, se realizó un catastro de las estructuras con daño estructural en la Provincia de Santiago, contabilizando un total de 10705 viviendas y 560 estructuras de dimensión mayor dañadas. En paralelo, se efectuó la caracterización de las condiciones geológicasgeotécnicas de la cuenca, considerando los siguientes factores: basamento rocoso, nivel freático, frecuencia fundamental y marco geológico. Conjuntamente, se estudia la influencia de antiguos cursos de agua que pueden haber generado depósitos de sedimentos sueltos, o blandos. Los resultados del análisis indican los mayores daños se produjeron en estructuras cimentadas sobre depósitos de suelos finos (unidad VII) y sobre depósitos de ceniza volcánica (unidad VI). Asimismo, se evidenció una cierta concentración de daños irreparables en la zona final de la lengüeta de la unidad IIIa (depósitos aluviales), caracterizada en su tramo final por depósitos de suelos finos

Palabras clave: efecto de sitio, amplificación sísmica, condiciones geológicas del terreno, daños sismo $27 \mathrm{~F}$
The $27 F$ earthquake of magnitude $M w=8.8$, affected a large area of Chile and left more than 2 million of homeless. In the metropolitan area, the most serious damage focused on older houses, however, significant damage was also presented in modern constructions. In order to identify the geological and geotechnical variables of the Santiago basin that influenced their seismic response, a survey of the distribution of the structures with damages in Santiago Province was performed, accounting for a total of 10705 houses and 560 larger structures damaged. In parallel, the characterization of geotechnical-geological basin conditions was performed including the following factors: depth of bedrock, water table, fundamental frequency and geological setting. Additionally, the influence of former waterways that may have generated deposits of loose or soft sediments was investigated. The results of the analysis indicated that major damage occurred in structures founded on fine soil deposits (unit VII) and volcanic ash deposits (unit VI). Also, a certain concentration of irreparable damage was evident in the end zone of unit IIIa (alluvial deposits), characterized in its final stretch by deposits of fine soils.

Keywords: site effect, seismic amplification, geological soils conditions, damages $27 \mathrm{~F}$ earthquake

\section{Introducción}

El mega terremoto del 27 de febrero del 2010 afectó a una importante parte del país, registrando daños que abarcaron transversalmente distintos tipos de estructuras, como viviendas, edificios de gran altura, hospitales, aeropuertos, iglesias y obras viales (puentes, rutas, pasos sobre nivel). A raíz de ello, y considerando que Chile se encuentra en un ambiente tectónico en permanente actividad, diversos grupos disciplinarios han realizado estudios de los posibles factores causantes de los daños, entre los cuales se distingue el fenómeno de amplificación sísmica del terreno. En este contexto y considerando que la cuenca de Santiago representa cerca del $40 \%$ de la población nacional (INE, 2002), es de vital importancia el reconocimiento y análisis 
de los factores que influyeron en su comportamiento sísmico. Consecuentemente, en este trabajo se analizan los daños ocurridos a través de un catastro realizado a las obras que presentaron daño estructural en las 32 comunas de la Región Metropolitana RM. En particular se analizó como posible causa de los daños, antiguos cursos de agua que podrían haber sido rellenados con materiales no controlados, o haber depositado sedimentos blandos o sueltos. Para ello se compararon las antiguas vías de aguas con las actuales, utilizando mapas históricos de la RM. Adicionalmente, se realizó la caracterización de las condiciones geológicas-geotécnicas de la cuenca de Santiago, que en definitiva podrían permitir explicar las concentraciones de daños observados en ciertos sectores. Los parámetros analizados son: profundidad del basamento rocoso, profundidad de la napa subterránea, frecuencia fundamental de los depósitos de suelos y geología.

\section{Catastro de daños generados por el terre- moto del 27F}

El catastro de obras con daños estructurales en la Provincia de Santiago fue hecho en base a los registros disponibles de las 32 municipalidades de la Provincia de Santiago. Los daños se dividieron en dos categorías dependiendo del tipo de edificación: Categoría A: correspondiente a las viviendas de uno o dos pisos. Se contabilizaron un total de 10705 viviendas con daños estructurales. Categoría B: correspondiente a toda estructura que no clasifique como categoría A, es decir, edificios, blocks habitacionales, hospitales, establecimientos educacionales, edificios patrimoniales o galpones. Se contabilizaron un total de 560 estructuras de dimensión mayor. Adicionalmente, se han identificado aquellas estructuras importantes que presentaron daño estructural severo. Estos daños son divididos en dos tipos: Tipo 1: Estructura Habitable sólo después de reparaciones y refuerzo estructural y Tipo 2: Estructura No habitable, no es posible rehabilitar el edificio.

En las Figuras 1a, 1b y 1c se presentan la distribución de los daños en todas las estructuras (Categoría A y B), los daños en estructuras de Categoría B y los daños severos, respectivamente. En la Figura 1c se presenta en negro las estructuras con daño Tipo 1 y en rojo las estructuras Tipo 2. Se desprende que los daños Tipo 1 se ubican en toda la zona central de la cuenca, mientras que los daños Tipo 2 se ubicaron mayoritariamente en el sector sur. En la Tabla 1 se detallan las estructuras que presentaron el daño estructural más severo (Tipo 2).

Con respecto al número de viviendas existentes en cada comuna, resultan con mayor porcentaje de daños las de Quinta Normal (5.9\%), seguido por Pedro Aguirre Cerda (4.6\%) y Cerro Navia (3.8\%). Respecto del número total de viviendas dañadas, Quinta Normal resulta con un $16.9 \%$ de los daños, Pedro Aguirre Cerda con un 12.3\% y Cerro Navia con un $12.8 \%$. Por otra parte, para los daños en estructuras de Categoría B, las comunas más dañadas fueron Pedro Aguirre Cerda con 86 estructuras dañadas, seguido por Providencia con 57 y Ñuñoa con 43 estructuras.
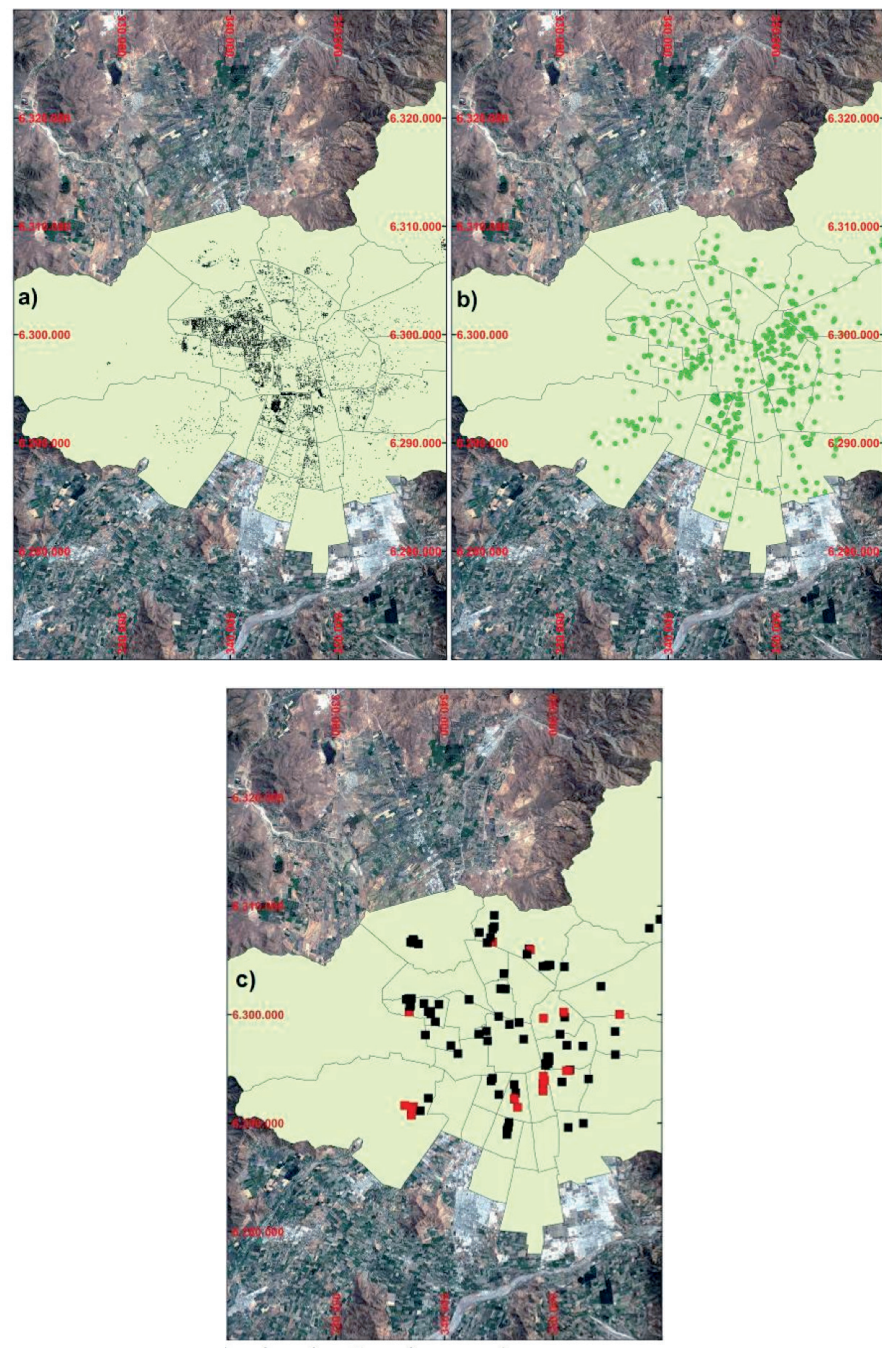

Figura 1: Ubicación de daños producto del 27F en la Provincia de Santiago, a) estructuras Categoría A y B, b) estructuras de Categoría B y c) estructuras con daños severos 
Tabla 1: Identificación de estructuras con daños severos

\begin{tabular}{|c|c|c|}
\hline Identificación & Ubicación & Observaciones \\
\hline Blocks habitacionales & $\begin{array}{l}\text { La Hondonada 8301, Cerro } \\
\text { Navia }\end{array}$ & Demolición del block producto de los daños \\
\hline Silo & $\begin{array}{l}\text { Santa Elena de Huechuraba } \\
1502 \text {, Huechuraba }\end{array}$ & Decreto de demolición producto de los daños \\
\hline $\begin{array}{l}\text { Municipalidad de Huechuraba, } \\
\text { Alcaldía }\end{array}$ & $\begin{array}{l}\text { Premio Nobel 5555, } \\
\text { Huechuraba }\end{array}$ & $\begin{array}{l}\text { Estructura presenta daños estructurales. Se decreta la demolición } \\
\text { del inmueble }\end{array}$ \\
\hline $\begin{array}{l}\text { Torres de Bilbao (losa } \\
\text { estacionamiento) }\end{array}$ & $\begin{array}{l}\text { Francisco Bilbao 8080, Las } \\
\text { Condes }\end{array}$ & $\begin{array}{l}\text { Colapso total de losa estacionamiento, destrucción de } 68 \\
\text { vehículos }\end{array}$ \\
\hline Edificio Sol Oriente I y II & $\begin{array}{l}\text { Macul } 2301 \text { y Exequiel } \\
\text { Fernández 2302, Macul }\end{array}$ & $\begin{array}{l}\text { Edificios de } 18 \text { pisos. Presentan graves daños estructurales, por } \\
\text { lo que se solicita su demolición }\end{array}$ \\
\hline $\begin{array}{l}\text { SMAPA Servicio Municipal de } \\
\text { Agua Potable y Alcantarillado }\end{array}$ & Alberto Llona 1806, Maipú & Daños estructurales en edificio, se decretó su demolición \\
\hline Don Luis Gandarillas 360 & Luis Gandarillas 360, Maipú & $\begin{array}{l}2 \text { torres de } 4 \text { pisos. Colapso de machones en subterráneo lo que } \\
\text { provocó el colapso del lado norte del edificio. }\end{array}$ \\
\hline $\begin{array}{l}\text { Condominio Don Tristán } \\
\text { Valdés }\end{array}$ & Tristán Valdés 216, Maipú & $\begin{array}{l}2 \text { torres de } 4 \text { pisos. Inclinación pronunciada y torsión del edificio } \\
\text { debido al desplomo de las columnas del poniente. }\end{array}$ \\
\hline $\begin{array}{l}\text { Condominio calle Hermanos } \\
\text { Carrera }\end{array}$ & $\begin{array}{l}\text { Hermanos Carrera 2727, } \\
\text { Maipú }\end{array}$ & $\begin{array}{l}\text { Torres de } 5 \text { pisos. Block poniente con fisuras diagonales en } \\
\text { muros. Block oriente con deformación de losas y grietas en } \\
\text { elementos estructurales. }\end{array}$ \\
\hline Copa de agua & Rafael Cañas 76, Providencia & $\begin{array}{l}\text { Estructura de soporte copa de agua colapsada. Decretada su } \\
\text { demolición }\end{array}$ \\
\hline Copa de agua & $\begin{array}{l}\text { Carlos Antúnez 2425, } \\
\text { Providencia }\end{array}$ & $\begin{array}{l}\text { Pilares que sustentan la copa de agua con hormigón colapsado y } \\
\text { enfierradura a la vista. }\end{array}$ \\
\hline $\begin{array}{l}\text { Conjunto habitacional Villa } \\
\text { Brasil }\end{array}$ & $\begin{array}{l}\text { José Mosqueira 3238, San } \\
\text { Joaquín }\end{array}$ & $\begin{array}{l}\text { Block con daños estructurales de consideración. Decretada su } \\
\text { demolición }\end{array}$ \\
\hline $\begin{array}{l}\text { Conjunto habitacional Villa El } \\
\text { Pinar }\end{array}$ & $\begin{array}{l}\text { Rio Palena 3670, San } \\
\text { Joaquín }\end{array}$ & $\begin{array}{l}\text { Block con daños estructurales de consideración. Decretada su } \\
\text { demolición }\end{array}$ \\
\hline $\begin{array}{l}\text { Conjunto habitacional Villa } \\
\text { Liszt }\end{array}$ & Liszt 3346, San Joaquín & $\begin{array}{l}\text { Block con daños estructurales de consideración. Decretada su } \\
\text { demolición }\end{array}$ \\
\hline Block habitacional & Liszt 3079, San Joaquín & $\begin{array}{l}\text { Daños estructurales de consideración en block. Decretada su } \\
\text { demolición }\end{array}$ \\
\hline Edificio & $\begin{array}{l}\text { Pasaje Blanco 5570, San } \\
\text { Miguel }\end{array}$ & Se decreta demolición producto de los daños \\
\hline Local comercial & $\begin{array}{l}\text { Gran Avenida 4836, San } \\
\text { Miguel }\end{array}$ & $\begin{array}{l}\text { Agrietamiento interior del } 2^{\circ} \text { piso, coronación superior del } \\
\text { edificio con riesgo de derrumbe hacia la vereda. }\end{array}$ \\
\hline
\end{tabular}

Características geológicas-geotécnicas de la cuenca y los daños ocurridos en el 27F

\section{Topografía superficial}

En la Figura 2a se presenta la elevación topográfica de la zona de estudio, observándose cambios de pendientes muy suaves, con elevaciones que descienden de este a oeste en franjas orientadas de norte a sur, desde los 1200 a $300 \mathrm{msnm}$. Los sectores de menor elevación son los ubicados en las comunas de Peñaflor y Talagante. La suave topografía es producto de la colmatación paulatina de sedimentos de origen fluvial, aluvial, lacustre y volcánicos provenientes principalmente del borde oriental de la cuenca. En la Figura $2 \mathrm{~b}$ se presentan los daños en todas las estructuras y en la Figura 2c los daños severos. En ambas figuras se observa que los daños se distribuyeron en distintas elevaciones topográficas y estos daños no poseen un patrón de elevación determinado.

\section{Basamento rocoso}

La morfología del basamento rocoso se ha basado en el modelo tridimensional realizado por Araneda et al. (2000) en la cuenca de Santiago, obteniéndose el mapa de profundidades de la Figura 3a. Se observa la irregularidad del basamento, con depresiones de más de 500 m y una serie de cerros islas de hasta $480 \mathrm{~m}$ de altura. A excepción de la 
alta concentración de daños en la comuna de Pudahuel (ver Figura $3 b$ ), justo donde la roca se encuentra más profunda, entre 450 a $500 \mathrm{~m}$, no se observa una concentración de daños en profundidades específicas.
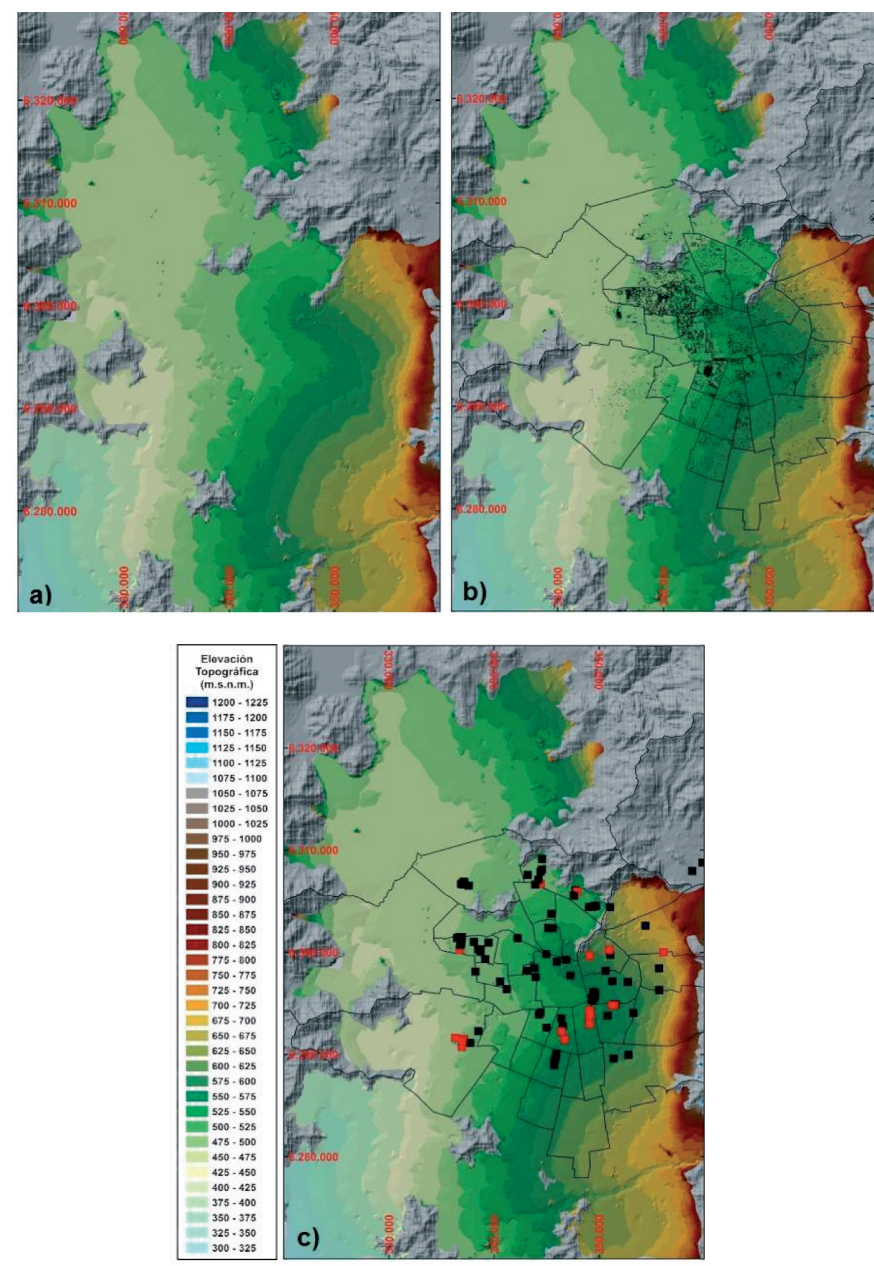

Figura 2: a) Elevación topográfica, b) daños estructuras categoría A y B y c) daños severos

\section{Napa subterránea}

Para la realización del mapa de aguas subterráneas en la zona de estudio, se consideró la información entregada por niveles estáticos de pozos de monitoreo reportados por la Dirección General de Agua DGA y el Banco Nacional de Aguas BNA. En la Figura 4a se presenta el mapa de profundidades del nivel freático en la cuenca de Santiago. Por un lado, los sectores con niveles freáticos más profundos se ubican al sureste de la cuenca con profundidades máximas entorno a los 150 metros. Las aguas subterráneas más superficiales se sitúan en la zona oeste, con profundidades mínimas entre 0 a $5 \mathrm{~m}$. Por su parte, en la Figura $4 \mathrm{~b}$ se presentan los daños en todas las



Figura 3: a) Profundidad del basamento rocoso, b) daños categoría $\mathrm{A}$ y $\mathrm{B}$ y c) daños severos

estructuras y en la Figura 4c los daños severos. En estas figuras se observa que no existe una concentración de daños a una profundidad determinada de las aguas subterráneas.

\section{Marco geológico}

En la Figura 5 se presenta el mapa geológico de la zona de estudio con la descripción de cada unidad geológica. Leyton et al. (2010) define 10 unidades geológicas que conforman el relleno de la cuenca de Santiago. Estas unidades fueron definidas utilizando la información obtenida de pozos y de los estudios desarrollados por Valenzuela (1978), Wall et al. (1999), Milovic (2000), Fernández (2001, 2003), Sellés y Gana (2001), Rauld (2002) y Fock (2005), considerando las principales características granulométricas de los primeros $30 \mathrm{~m}$ de profundidad. En la Figura 6a se presentan los daños en todas las estructuras y en la Figura $6 \mathrm{~b}$ los daños severos (en estructuras de categoría B Tipo 1 y 2), emplazadas sobre las 10 unidades geológicas de la cuenca. 



Figura 7: Pendientes de la topografía superficial de la cuenca desde E: 340.000 a 360.000 UTM

falla geológica Infiernillo-Cerro Renca-Portezuelo de Chada ubicada en la zona oeste de la cuenca, tal como se muestra en la Figura 8a. Esta falla pone en contacto a las rocas mesozoicas de la Formación Lo Valle con las rocas cenozoicas de la Formación Abanico. Esta estructura fue descrita por Sellés y Gana (2001) como una falla normal invertida. Cabe mencionar que la traza de la falla Infiernillo-Cerro Renca-Portezuelo de Chada corresponde a una interpretación realizada considerando los contactos geológicos y las edades de los afloramientos rocosos estimados de algunos sectores. Por lo tanto, su trazado podría ser redefinido al presentado en línea punteada azul en la Figura 8b, considerando la diferencia de daños entre el lado oeste y este de la senda. En la zona noroeste de la falla se observa una importante cantidad de daños, no obstante esto podría deberse a que los daños se emplazan sobre la unidad VI y VII. El resto de los daños, como los observados en la unidad II, obedecerían a otros factores no sólo a la geología del sector.

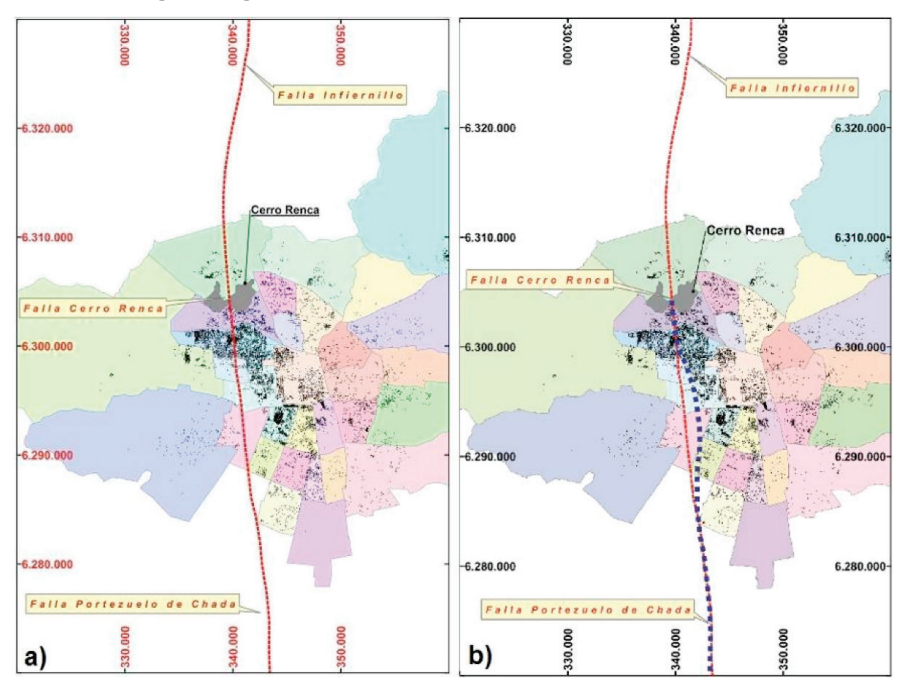

Figura 8: a) Falla Infiernillo-Cerro Renca-Portezuelo de Chada y b) reinterpretación traza de falla

\section{Antiguos cursos de aguas superficiales}

El depósito paulatino de suelos finos a través del transporte de material en suspensión, proveniente de ríos, esteros y canales, puede generar un relleno de materiales de baja consistencia o compacidad en sus planicies adyacentes. Antiguos cauces podrían haber sido rellenados sin control y corresponder a singularidades de mayor demanda sísmica. En este contexto, se decidió investigar si la presencia de estos sedimentos sueltos fue un factor influyente en los daños provocados por el terremoto del 27F. En la Figura 9a se presenta el mapa con los cursos de aguas naturales y artificiales. Los cursos artificiales son trazados en color rojo, mientras que los antiguos cursos naturales en color negro y los cursos naturales actuales en azul.

En la Figura 9b se presentan los daños en todas las estructuras y en la Figura 9c los daños severos. Se observa que los daños generales se concentrarían en primera instancia en dos zonas (A y B) y los daños severos podrían ser agrupados en las zonas 1, 2 y 3 . No obstante, a excepción de pequeñas concentraciones de daños alrededor del Zanjón de La Aguada y el canal San Joaquín, es posible que los daños puedan ser más bien asociados a otros parámetros como la geología local. 

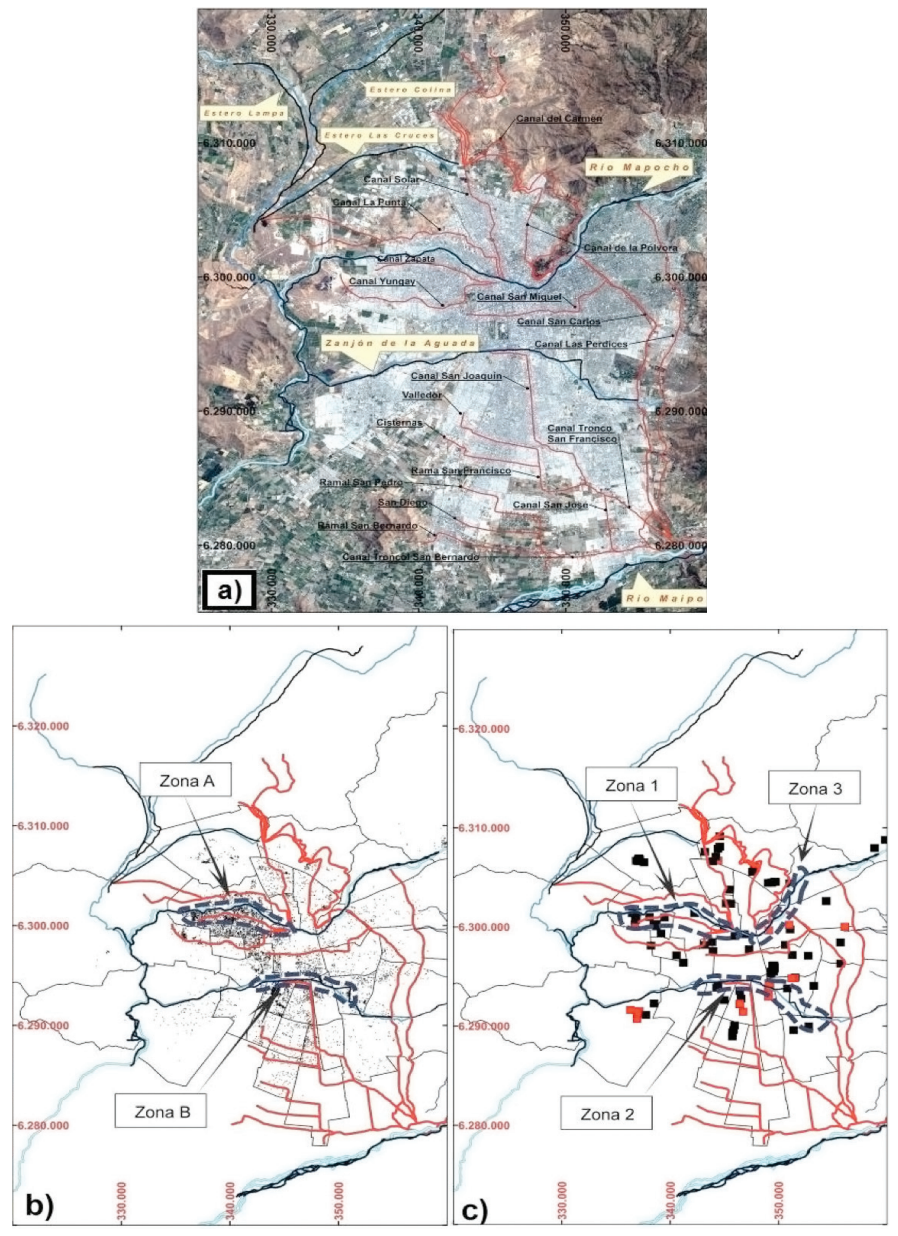

Figura 9: a) Antiguos cursos de agua, b) daños en todas las estructuras y c) daños severos

\section{Frecuencia preponderante}

Se utilizó el estudio de la respuesta sísmica de la cuenca de Santiago mediante el método de Nakamura desarrollado por Pastén (2007). En la Figura 10a se presenta la distribución de las frecuencias preponderantes de vibración emplazadas sobre la geología y la profundidad del basamento rocoso. Se observa que las zonas de bajas frecuencias (menores a $0.5 \mathrm{~Hz}$ ), se presentan en tres sectores de la cuenca: en la zona norte alrededor del cerro Renca en comunas como Pudahuel y Cerro Navia, al este de la cuenca, en las comunas como de Ñuñoa y Peñalolén; y al sur del cerro Chena. Por su parte, las frecuencias entre 0.5 a $2 \mathrm{~Hz}$ se distribuyen en toda la cuenca y las zonas de mayores frecuencias, entre 5 a $10 \mathrm{~Hz}$, se centran en la zona este de la línea imaginaria que une el cerro Renca y el cerro Chena. Adicionalmente, se observa que en la zona noroeste de la cuenca, constituida por suelos finos y cenizas volcánicas, se registran frecuencias fundamentales menores a $2 \mathrm{~Hz}$, y consistentemente, en esa zona, los sectores donde el basamento rocoso es más profundo, las frecuencias registradas fueron las más bajas $(0.5 \mathrm{~Hz})$. Por su parte, a pesar de que en las zonas con sedimentos más rígidos (unidad II) se registraron frecuencias altas, también se presentaron frecuencias bajas, incluyendo los sectores donde las rocas no son tan profundas. Lo anterior indicaría que existen depósitos de suelos que no son considerados en la geología superficial de la cuenca, o que existe una configuración geotécnica de sedimentos en profundidad compleja.

Con respecto a los daños en todas las estructuras, como se muestra en la Figura 10b se observa que dichos daños se concentraron mayoritariamente, en el caso de las viviendas, próximos a frecuencias fundamentales menores a $2 \mathrm{~Hz}$. Respecto a las estructuras con daños severos estás se ubicaron cercanos a frecuencias fundamentales variables entre 0 a $10 \mathrm{~Hz}$.
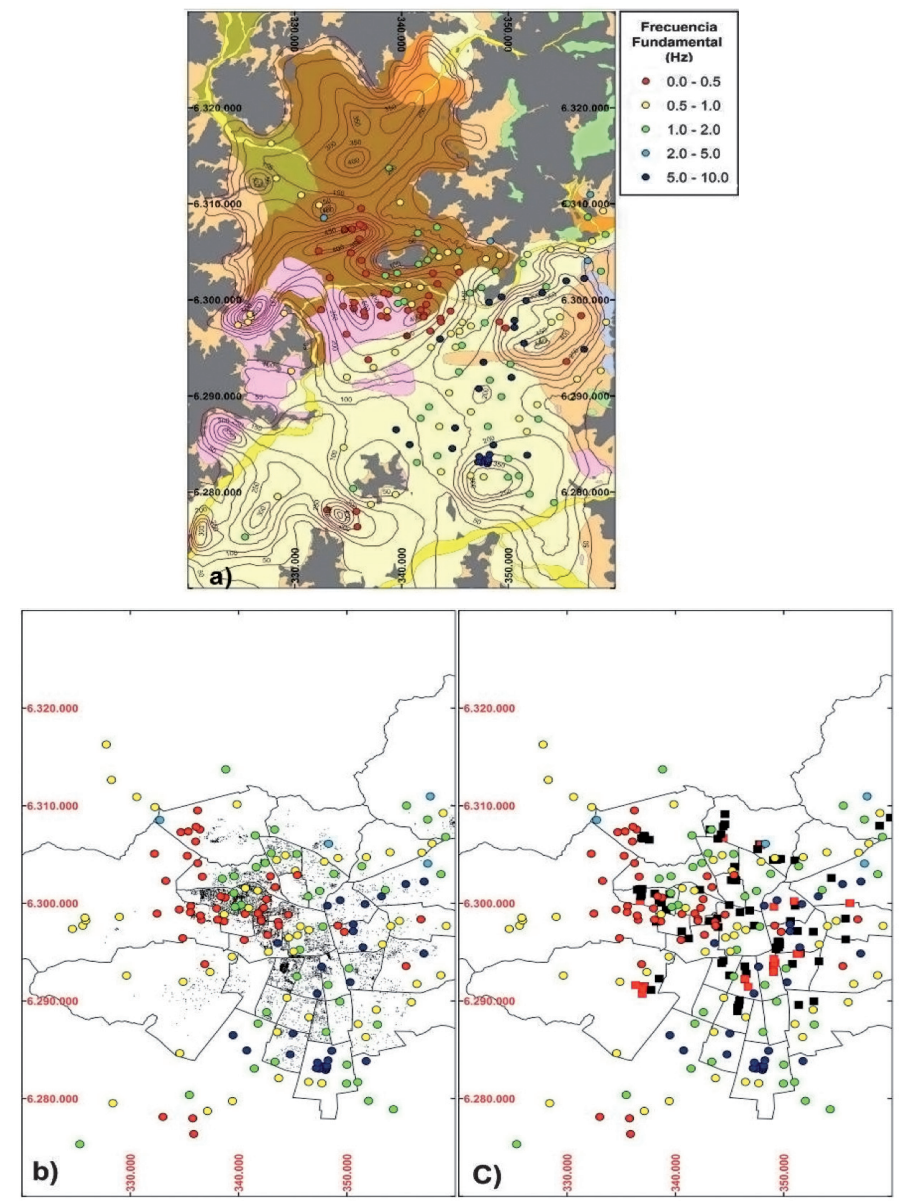

Figura 10: a) Frecuencias fundamentales, b) daños estructuras categoría A y B y c) daños severos 


\section{Conclusiones}

De acuerdo a los resultados obtenidos, resulta posible plantear las siguientes conclusiones:

Parámetros de la cuenca de Santiago, como profundidad del nivel freático, topografía de superficie, distribución de frecuencias de vibración preponderantes y profundidad del basamento rocoso, en forma individual, no fue posible correlacionarlos directamente con la distribución de los daños observados.

Respecto a la geología superficial de la cuenca, los mayores daños se ubicaron sobre depósitos de suelos finos (unidad VII) y ceniza volcánica (unidad VI). Asimismo, se evidenció una concentración de daños irreparables en la zona final de la lengüeta de la unidad IIIa, caracterizada en su tramo final por depósitos de suelos finos. Por lo tanto, se propone que la unidad IIIa abarcaría una extensión mayor hacia el sur de la cuenca. En consecuencia, se concluye que los depósitos de suelos finos y ceniza volcánica generaron una amplificación sísmica con una mayor demanda a las estructuras y por consiguiente, provocando un daño mayor. Adicionalmente, algún grado de correlación se observa entre la concentración de daños y algunos cauces de aguas superficiales como el Zanjón de La Aguada y el Canal San Joaquín. No obstante, podría deberse a la extensión de la lengüeta IIIa.

Por otro lado, se puede identificar una diferencia importante de daños al este y oeste de la falla del cerro Renca, cuyo trazado podría re-interpretarse más al este, siendo así posible explicar la diferencia de daños, debido a que el basamento rocoso al este y oeste de la falla pertenecen a formaciones geológicas distintas, y por tanto, existiría una diferencia de rigidez que podría ser la responsable de los daños. Esta hipótesis requiere en definitiva mayor investigación.

\section{Agradecimientos}

Los autores agradecen a las 32 Municipalidades de la Provincia de Santiago por la entrega de información con la cual se pudo realizar el catastro de 11265 obras con daños estructurales producto del 27F. Asimismo, agradecer a la Biblioteca Nacional por su ayuda en encontrar antiguos mapas de la Región Metropolitana.

\section{Referencias}

Araneda, M., Avendaño, M. y Merlo, C. (2000). Modelo gravimétrico de la cuenca de Santiago, etapa III final. IX Congreso Geológico de Chile, Puerto Varas, Chile, 2, 404-408

Fernández, J.C. (2003). Respuesta sísmica de la cuenca de Santiago. Servicio Nacional de Geología y Minería. Carta Geológica de Chile. Serie Geología Ambiental Nº1.

Fernández, J.C. (2001). Estudio geológico-ambiental para la planificación territorial del sector Tiltil - Santiago. Memoria de título, Universidad de Chile

Fock, A. (2005). Cronología y tectónica de la exhumación en el Neógeno de Los Andes de Chile Central entre los $33^{\circ}$ y los $34^{\circ}$ S. Tesis de Magíster, Universidad de Chile.

INE Y CELADE (2002). División de Población de la CEPAL e Instituto Nacional de Estadísticas de Chile INE. Chile Censo de Población y Vivienda 2002

Leyton, F., Sepúlveda, S., Astroza, M., Rebolledo, S., González, L., Ruiz, R., Foncea, C., Herrera, M. y Lavado, J. (2010). Zonificación sísmica de la cuenca de Santiago. X Congreso Chileno de Sismología e Ingeniería Antisísmica. ACHISINA, Santiago

Milovic, J.J. (2000). Estudio geológico-ambiental para el ordenamiento territorial de la mitad sur de la cuenca de Santiago. Memoria de título, Universidad de Concepción

Nichols, G. (2009). Sedimentology and Stratigraphy. Second Edition. Wiley - Blackwel, Oxford

Pastén, C. (2007). Respuesta sísmica de la cuenca de Santiago. Tesis de Magíster, Universidad de Chile

Rauld, R. (2002). Análisis morfoestructural del frente cordillerano Santiago Oriente, entre el río Mapocho y quebrada de Macul. Memoria de título, Universidad de Chile

Sellés, D. y Gana, P. (2001). Geología del área de Talagante-San Francisco de Mostazal, regiones Metropolitana de Santiago y del Libertador General Bernardo O'Higgins. Servicio Nacional de Geología y Minería. Carta Geológica de Chile. Serie Geológica Básica, N74

Valenzuela, G. (1978). Suelo de Fundación de Santiago. Instituto de Investigaciones Geológicas. Boletín N³3

Wall, R., Sellés, D. y Gana, P. (1999). Área Tiltil-Santiago, Región Metropolitana. Servicio Nacional de Geología y Minería. Mapas Geológicos $\mathrm{N}^{\circ} 11$ 